\title{
Elemental abundances and temperatures of quiescent solar active region cores from X-ray observations ${ }^{\star}$
}

\author{
G. Del Zanna and H. E. Mason
}

\begin{abstract}
DAMTP, Centre for Mathematical Sciences, Wilberforce Road, Cambridge CB3 0WA, UK
e-mail: g.del-zanna@damtp.cam.ac.uk
\end{abstract}

Received 20 January 2014 / Accepted 18 March 2014

\section{ABSTRACT}

\begin{abstract}
A brief review of studies of elemental abundances and emission measures in quiescent solar active region cores is presented. Hinode EUV Imaging Spectrometer (EIS) observations of strong iron spectral lines have shown sharply peaked distributions around 3 MK. EIS observations of lines emitted by a range of elements have allowed good estimates of abundances relative to iron. However, X-ray observations are required to measure the plasma emission above $3 \mathrm{MK}$ and the abundances of oxygen and neon. We revisit, using upto-date atomic data, older X-ray observations obtained by a sounding rocket and by the Solar Maximum Mission (SMM) Flat Crystal Spectrometer (FCS). We find that the $\mathrm{Fe} / \mathrm{O}$ and $\mathrm{Fe} / \mathrm{Ne}$ ratios are normally increased by a factor of 3.2, compared to the photospheric values. Similar results are obtained from FCS observations of six quiescent active region cores. The FCS observations also indicate that the emission measure above $3 \mathrm{MK}$ has a very steep negative slope, with very little plasma observed at $5 \mathrm{MK}$ or above.
\end{abstract}

Key words. Sun: abundances - Sun: corona - Sun: X-rays, gamma rays - techniques: spectroscopic

\section{Introduction}

This paper discusses measurements of elemental abundances and temperatures in the hot active region (AR) cores obtained from remote-sensing instruments. By "AR cores" we mean the hot loops that typically have temperatures of $3 \mathrm{MK}$, hence are very bright in emission lines formed at this temperature, in particular in the X-rays. Previous measurements are briefly reviewed, and some $\mathrm{X}$-ray observations revisited, using up-to-date atomic data. Measurements of elemental abundances and temperatures are closely linked, since the differential emission measure (DEM) distribution is used to both describe the temperature distribution of the plasma and obtain relative elemental abundances.

It is well known that solar coronal abundances present variations and differ from the photospheric ones. The ratio of the abundances of the low $(\leq 10 \mathrm{eV})$ first ionisation potential (FIP) elements vs. the high-FIP ones is higher in coronal plasma, however the absolute abundances (relative to hydrogen) are still debated, as well as the amplitude of the difference. For recent reviews on the issue of solar abundances see, e.g., Asplund et al. (2009), Lodders et al. (2009), Schmelz et al. (2012).

There is a vast literature on the subject of solar coronal abundances, but comparatively little related to active region (AR) cores. In fact, most published results on chemical abundances are based on either observations with uncollimated spectrometers (often full Sun), or flare observations.

In terms of AR core temperature measurements, there is a vast literature. Rosner et al. (1978) found that Skylab X-ray imaging was consistent with the hot core loops in quiescent ARs being approximately isothermal around $3 \mathrm{MK}$, an observational aspect that was found puzzling. Early X-ray spectroscopy also found a similar result (Evans \& Pounds 1968).

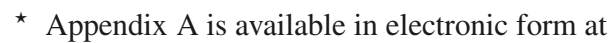
http://www . aanda.org
Later spectroscopic measurements in the X-rays from the Solar Maximum Mission (SMM) Flat Crystal Spectrometer (FCS) of many ARs have often shown that little material at temperatures above $3 \mathrm{MK}$ is present, and therefore most of the abundance analysis have assumed that the plasma was effectively isothermal (see, e.g. Schmelz et al. 1996).

Extreme ultraviolet (EUV) spectroscopy with the SOHO Coronal Diagnostic Spectrometer (CDS; Mason et al. 1999; Del Zanna \& Mason 2003) and Hinode EUV Imaging Spectrometer (EIS; O'Dwyer et al. 2011; Tripathi et al. 2011; Winebarger et al. 2011; Warren et al. 2012; Schmelz \& Pathak 2012; Del Zanna 2013b) have indicated that the plasma distribution in the $1-3 \mathrm{MK}$ range is very steep, and that little material is present at higher temperatures, although this issue is still debated in the literature.

The EUV measurements such as those from Hinode/EIS constrain very well the plasma distribution up to $3 \mathrm{MK}$, but are limited at higher temperatures, because hotter lines are normally weak and/or blended. For a discussion of the Hinode EIS Fe XVII lines see Del Zanna \& Ishikawa (2009), while details on the hotter Hinode EIS lines have been given e.g. in Young et al. (2007), Del Zanna (2008a, 2012, 2013b), Warren et al. (2008); Ko et al. (2009), Del Zanna et al. (2011b).

As discussed in Del Zanna (2013b), previous studies that did not consider the in-flight Hinode EIS calibration (Del Zanna 2013a) underestimated by a factor of two the intensities in lines formed around $3 \mathrm{MK}$, hence the actual steepness of the EM distribution of the active region cores was underestimated.

Assessing the temperature distribution of the AR core hot plasma above $3 \mathrm{MK}$ is an important constraint to nanoflare models (see, e.g. Patsourakos \& Klimchuk 2009; Bradshaw \& Klimchuk 2011, and references therein). There is ample literature on measurements of this hot component in active regions, using broad-band X-ray observations from e.g. Yohkoh SXT and Hinode XRT (see, e.g. references in Reale 2010). However, 
Table 1. Active region abundance measurements relative to iron.

\begin{tabular}{lclllllllllll}
\hline \hline & & & & & & & & & \multicolumn{3}{c}{ Photospheric } & \multicolumn{2}{c}{ Coronal } \\
$\mathrm{Fe} / \mathrm{El}$. & (FIP) & E68 & D75 & E & P77 & W94 & M94 & D13 & Present & A09 & F92 & S12 \\
\hline $\mathrm{Fe} / \mathrm{Ne}$ & $(21.6)$ & 2.5 & 1.7 & - & 1.0 & 1.1 & - & - & 1.2 & $0.37^{\dagger}$ & 1.05 & 0.88 \\
$\mathrm{Fe} / \mathrm{Ar}$ & $(15.8)$ & - & - & 13.2 & - & - & 25 & 50 & - & $12.6^{\dagger}$ & 33 & 31 \\
$\mathrm{Fe} / \mathrm{O}$ & $(13.6)$ & 0.28 & 0.26 & - & 0.16 & 0.16 & - & - & 0.2 & 0.065 & 0.16 & 0.17 \\
$\mathrm{Fe} / \mathrm{S}$ & $(10.4)$ & - & - & - & - & - & 4.0 & 6.8 & - & 2.4 & 6.8 & 4.2 \\
$\mathrm{Fe} / \mathrm{Si}$ & $(8.1)$ & - & - & - & - & - & - & 1.0 & - & 1.0 & 1.0 & 0.98 \\
$\mathrm{Fe} / \mathrm{Mg}$ & $(7.6)$ & - & - & - & 1.0 & 1.05 & - & 0.8 & - & 0.8 & 0.9 & 0.96 \\
$\mathrm{Fe} / \mathrm{Ni}$ & $(7.6)$ & 6.0 & - & - & 17.8 & - & 8.3 & 29.5 & - & 19.1 & 18.2 & 19.9 \\
$\mathrm{Fe} / \mathrm{Ca}$ & $(6.1)$ & - & - & 11.2 & - & - & - & 13.5 & - & 14.5 & 14.8 & 16.2 \\
\hline
\end{tabular}

Notes. The abundances are listed with decreasing FIP of the element (eV, the FIP of Fe is 7.9). Previous values from quiescent active region observations: E68: Evans \& Pounds (1968); D75: Davis et al. (1975); E: Mason (1975) for Ca and Young et al. (1997) for Ar; P77: Parkinson (1977); W94: Waljeski et al. (1994); M94: Monsignori Fossi et al. (1994); D13: Del Zanna (2013b), obtained from Hinode EIS in the EUV. For reference, we show the Asplund et al. (2009) [A09] photospheric abundances ( ${ }^{\dagger}$ the Ne and Ar values are not obtained from photospheric measurements) and the generic coronal abundances of Feldman et al. (1992) [F92] and Schmelz et al. (2012) [S12].

these instruments are broad-band and the results are therefore uncertain.

Fe XVIII emission is often observed in ARs with the Solar Dynamic Observatory (SDO) Atmospheric Imaging Assembly (AIA, see Lemen et al. 2012) $94 \AA$ band, so it has been used to provide a further constraint on the emission measure (EM) above 3 MK. For example, Warren et al. (2012) combined Hinode EIS with AIA $94 \AA$ observations to find the slope $\beta$ of the distribution $\left(\mathrm{EM}=T^{-\beta}\right)$ in the $3-10 \mathrm{MK}$ range. The $\beta$ values ranged between 6 and 10 .

However, as shown in Del Zanna (2013b), a considerable part of the Fe XVIII emission can come from the 3 MK plasma and the $94 \AA$ band is highly multi-thermal, so it is very easy to overestimate the Fe XVIII emission. A proper assessment of the plasma at temperatures higher than $3 \mathrm{MK}$ requires observations in the X-rays.

The first observations of active region cores were carried out during total solar eclipses. The active region cores were called coronal condensations. We searched the literature of eclipse observations and found that the only clear and well-calibrated observation of a coronal condensation was done in 1952 in Kharthoum, as described by Aly et al. (1962). The coronal condensation that was observed was unusually bright and a series of calcium (including the hot $\mathrm{CaXV}$ ) and iron lines were observed. The continuum was also observed, so in principle these are excellent observations to constrain the absolute abundance of iron and calcium, as well as the temperature. The interpretation of the line and continuum emission is not straightforward though, and requires some modelling and some assumptions on the emitting source. This was done by Mason (1975), where the calcium abundance was found to be 6.8 dex and the iron abundance 7.85 dex (in the usual logarithmic notation where the hydrogen abundance is 12), i.e. an enhancement of about a factor of three for both elements, relative to their photospheric values. We also note that Young et al. (1997) reviewed the original measurements by Aly et al. (1962) of an Ar XIV forbidden line to find an $\mathrm{Ar} / \mathrm{Ca}$ relative abundance of $0.85 \pm 0.20$.

With the advent of the space observations, an extensive literature on EUV measurements has appeared, however very few abundance measurements pertained to AR cores. In fact, many observations in the EUV either used emission lines formed around or below $1 \mathrm{MK}$, hence probed different AR structures, or compared emission lines formed over very different temperatures, hence likely formed in spatially different regions. One example is the paper on the OSO-6 observations by Withbroe (1975). A significant number of results pertain to either flare spectra or the legs of warm loops, i.e. only point-like features (as observed e.g. with the Skylab NRL S082A EUV spectra, see Widing \& Feldman 1993).

A sample of abundance measurements of active region cores that are relevant for this paper are shown in Table 1. Monsignori Fossi et al. (1994) used SERTS EUV observations of an AR core to obtain, with a DEM analysis, relative elemental abundances for many elements. The DEM presented the usual two-peak structure, with one peak at $1 \mathrm{MK}$ due to the overlying corona, and one at $3 \mathrm{MK}$ due to the core of the AR. Lines from $\mathrm{S}, \mathrm{Ni}$, Ar, Fe formed around $3 \mathrm{MK}$ were observed. The following abundances were obtained: $\mathrm{Fe} / \mathrm{S}=4, \mathrm{Fe} / \mathrm{Ni}=8.3, \mathrm{Fe} / \mathrm{Ar}=25$.

The SOHO SUMER spectrometer carried out limited observations of ARs in the UV. Mohan et al. (2000) used the observed intensities of lines from Si XI, K XIII, S X, and Ar XII to study the relative abundances of $\mathrm{Si}, \mathrm{K}, \mathrm{S}$, and $\mathrm{Ar}$ (two low-FIP and two high-FIP elements). The SUMER observations scanned an AR off-limb. The S/Ar abundance was found to be nearly constant with height at around $4.5-5$, i.e. close to its "photospheric" value recommended by Asplund et al. (2009), 5.2. The Si/Ar abundance was found to decrease with height, while the K/Ar was found to increase, a puzzling result. Both $\mathrm{Si} / \mathrm{Ar}$ and $\mathrm{K} / \mathrm{Ar}$ relative abundances were higher than the photospheric values.

That active region cores were more consistent with a FIP enhancement of 4 rather than photospheric abundances was previously noted (see, e.g. Tripathi et al. 2011). However, the first comprehensive measurement of the abundances of several elements in an AR core was recently published in Del Zanna (2013b), using Hinode EIS observations and a recent calibration (Del Zanna 2013a). The relative abundances of several key elements were obtained, including low-FIP (e.g. Fe, Si, Ca, Ni) and high-FIP (e.g. Ar, S). A FIP enhancement of 3.2 was found. Other active region cores showed similar abundances. A consideration of the path length suggested that the iron absolute abundance must be enhanced, compared to its photospheric value, by at least a factor of three. The $\mathrm{Ca}$ and $\mathrm{Ar}$ abundances, relative to iron, were well constrained, and were $7.4 \times 10^{-6}$ and $2 \times 10^{-6}$. The line-to-continuum measurements of the $\mathrm{Ca}$ abundance by Mason (1975) was $6.3 \times 10^{-6}$, in excellent agreement, while the Ar abundance of $5.4 \times 10^{-6}$ by Young et al. (1997) is significantly higher. 
One limitation of the EUV observations is the inability to constrain the relative abundances of oxygen and neon, two important high-FIP elements. X-ray observations are needed for this, due to the presence of the strong resonance lines from O VIII $19 \AA$ and Ne IX $13.4 \AA$.

An early measurement of the AR abundances of oxygen and neon using X-ray spectroscopy was obtained by Evans \& Pounds (1968) with a rocket spectrum. They found the AR core to be nearly isothermal at $3 \mathrm{MK}$, and measured the relative abundances between iron, oxygen and neon. They obtained $\mathrm{Fe} / \mathrm{O}=$ 0.28 and $\mathrm{Fe} / \mathrm{Ne}=2.5$. Davis et al. (1975) also used a rocket $\mathrm{X}$-ray spectrum (with a collimator) to obtain $\mathrm{Fe} / \mathrm{O}=0.26$ and $\mathrm{Fe} / \mathrm{Ne}=1.7$. One of the best X-ray spectra of a quiescent AR was obtained by Parkinson (1975). Parkinson (1977) used these AR observations to obtain, using essentially a line ratio method, the relative abundances of a few elements. Fixing the abundance of $\mathrm{Si}$ to $7.65 \mathrm{dex}$, he measured the abundances of $\mathrm{O}(8.45)$, $\mathrm{Ne}$ (7.65), $\mathrm{Na}$ (6.5), $\mathrm{Mg}$ (7.65), $\mathrm{Fe}$ (7.65) and $\mathrm{Ni}(6.4)$, i.e. found relative abundances between iron, oxygen and neon that were quite different $(\mathrm{Fe} / \mathrm{O}=0.16$ and $\mathrm{Fe} / \mathrm{Ne}=1.0)$ from the previous measurements.

Excellent X-ray spectra of ARs were later obtained by the SMM FCS. There are several observations of the cores of quiescent ARs. The intensities of the strong lines were found by various authors (see, e.g. Waljeski et al. 1994) to be generally consistent with an FIP enhancement of a factor of $3.5(\mathrm{Fe} / \mathrm{O}=0.16$ and $\mathrm{Fe} / \mathrm{Ne}=1.12$ ), as suggested by the compilation of Meyer (1985).

However, large variations in the relative abundances of low-FIP vs. high-FIP elements as measured from SMM/FCS have been reported by several authors, for example Saba \& Strong (1993), Schmelz et al. (1996). Schmelz et al. (1996) performed a review of several SMM/FCS quiescent AR observations, and used the EM loci method to find large variations in the Ne abundance.

McKenzie \& Feldman (1992) also found a large scatter of $\mathrm{Fe} / \mathrm{Ne}$ and $\mathrm{Fe} / \mathrm{O}$ values, indicative of elemental abundance variations $(\mathrm{Fe} / \mathrm{Ne}$ ranged between 0.3 and 1.1, while the $\mathrm{Fe} / \mathrm{O}$ varied between 0.04 and 0.1 ). These authors studied the intensity ratios of the O VIII $19 \AA$, Ne IX $13.4 \AA$, Fe XVII $15.0 \AA$, and Fe XVIII $14.2 \AA$ observed by the SOLEX B spectrometer on board the P78-1 satellite in active regions. Their analysis did not take into account the differences in the temperature response of these ions though.

There are various reasons to revisit the previous results obtained from the X-ray observations. First, new atomic data have been made available. The strongest Fe XVII and Fe XVIII lines notoriously showed large (factors of 2-3) discrepancies between observed and predicted intensities. It turned out that the problems were largely due to the limitation of distorted wave (DW) calculations, as shown in Del Zanna (2011, 2006). Indeed, we found excellent agreement between solar observations and theory when $R$-matrix calculations (Liang \& Badnell 2010; Witthoeft et al. 2006) ${ }^{1}$ are used. We note, however, that many previous abundance studies used the strongest Fe XVII and Fe XVIII resonance lines, for which the predicted line intensities with the new atomic data changed by small amounts (typically 10-20\%). The really major difference in predicted intensities comes from recent calculations of ionisation and recombination rates. The latest comprehensive set of rates produced ion fractions (made available within CHIANTI v.6, see Dere et al. 2009)

\footnotetext{
1 Work done within the UK APAP network, see http://www. apap-network.org
}

that are significantly shifted in temperature compared to previous calculations such as those of Arnaud \& Rothenflug (1985), which were extensively used in the previous studies. For Fe XVII, the combined changes in the atomic data produces well over a factor of two difference in the emissivity at the peak ion abundance, which is indeed significant.

In terms of measuring abundances, it is useful to revisit older observations because the standard photospheric abundances of many elements have recently changed significantly, and because most previous studies made the assumption that the AR cores are isothermal. This is a reasonable assumption, although we note that any small departures could enhance the different temperature response of the various ions, leading to incorrect results.

In the next two sections we present a discussion of the best $\mathrm{X}$-ray measurements of AR cores we have available, while in the last section we draw our conclusions.

\section{Parkinson (1975) AR spectrum}

An instrument, built by the University of Leicester (UK), consisting of Bragg crystal spectrometers with a collimator was flown on a British Skylark sounding rocket on 30 Nov. 1971 and recorded the best X-ray solar spectrum of a quiescent active region (Parkinson 1975). The observations obtained with the KAP crystal in the 9-23 $\AA$ range are used here. The instrument had an excellent spectral resolution, was radiometrically calibrated and the whole spectral region was observed simultaneously, unlike many other X-ray instruments which scanned the wavelengths (e.g. SMM/FCS, see below). The Fe XVII lines, the Helike Ne IX $13.45 \AA\left(1 s^{2}-1\right.$ s $\left.2 p\right)$, the H-like Ne X $12.1 \AA(1 s-2 p)$, the O VII, O VIII were all prominent. The strongest Fe XVIII line, at $14.20 \AA$, was barely visible. Lines formed at higher temperatures were totally absent, indicating a lack of plasma with $T>4 \mathrm{MK}$.

One simple way to estimate if the plasma is nearly isothermal is the EM loci method, by which the curves $I_{\mathrm{ob}} /(A \times G(T))$ as a function of temperature are plotted $\left(I_{\mathrm{ob}}\right.$ is the observed intensity, $A$ the element abundance and $G(T)$ the contribution function containing the atomic parameters). If the curves cross at one temperature, then the plasma can be modelled with an isothermal distribution. In general, the loci of the curves represent upper limits to the emission measure distribution. For details, see Strong (1978) and Del Zanna et al. (2002).

Del Zanna (2011) applied the EM loci method to find a nearisothermal distribution for the Parkinson (1975) data and to show that the Fe XVII lines, in conjunction with $R$-matrix calculations (Liang \& Badnell 2010), offer an excellent diagnostic to measure the electron temperature. For the first time, excellent agreement between the observed and predicted Fe XVII intensities for the strongest lines was found, showing the potential of these lines for measuring temperatures. A value around $\log T[\mathrm{~K}]=6.5$, i.e. $3 \mathrm{MK}$ was found. The relative $\mathrm{Fe} / \mathrm{Ne}$ abundance needed to be increased by about a factor of two, compared to the recommended photospheric abundances of Asplund et al. (2009).

To obtain a proper measurement of the elemental abundances, we have performed a DEM analysis using the method described in Del Zanna (1999). The latest v.7.1 CHIANTI atomic data and ionisation equilibrium tables (Landi et al. 2013) are adopted here. We converted the Parkinson (1975) intensities assuming a collimator angular (FWHM) dimension of $3^{\prime}$.

We obtained the results shown in Fig. 1 and Table 2. Excellent agreement (within a relative 20\%) between observed and predicted radiances is found for all the strongest lines, with 

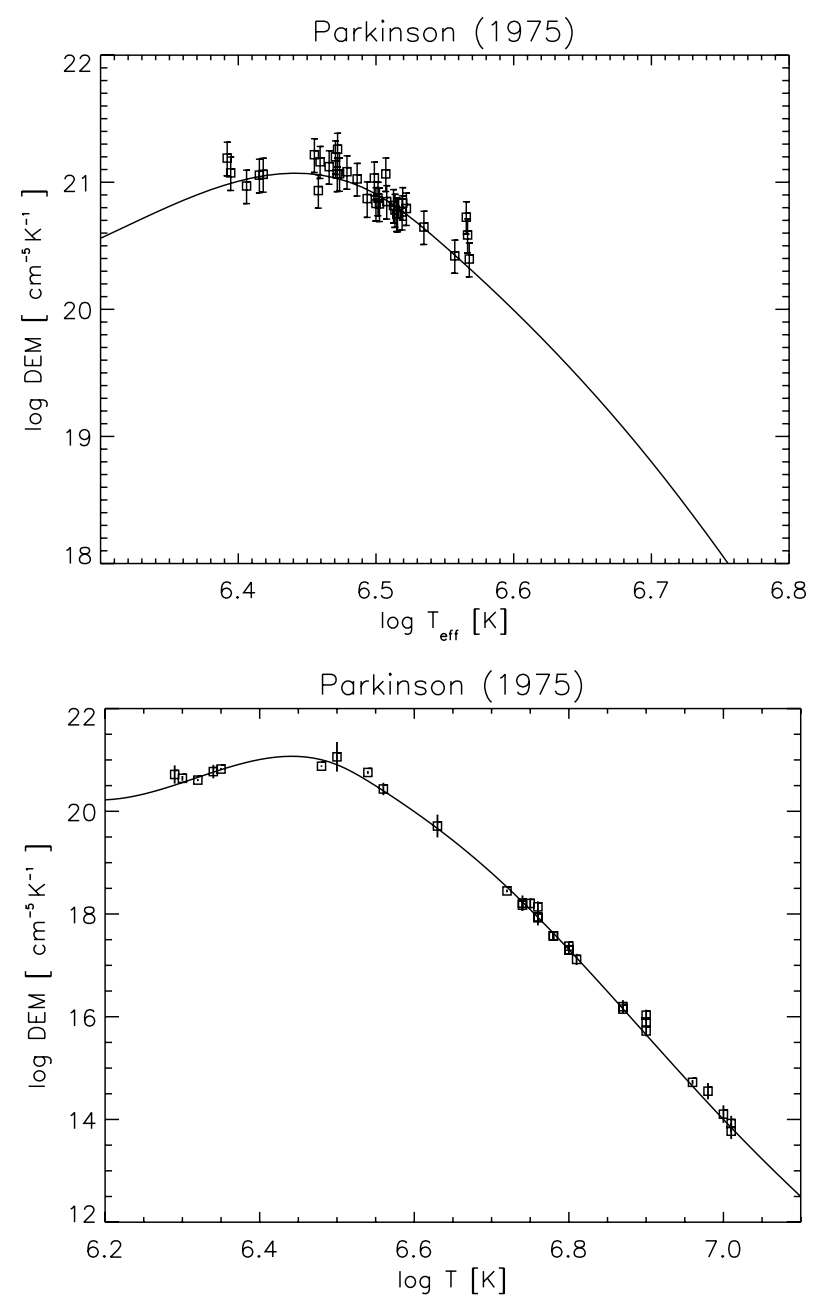

Fig. 1. Top: DEM for the Parkinson (1975) active region spectrum and the present elemental abundances. The ion and the wavelength $(\AA)$ is given. The numbers in parentheses are the ratios between predicted and observed intensities. The points are plotted at the effective temperature $T_{\text {eff }}$, and at the theoretical vs. the observed intensity ratio multiplied by the DEM value. Bottom: same DEM, but with the points plotted at the temperature of the maximum of the $G(T)$.

the elemental abundances shown in Table 1, where the lowFIP Fe and $\mathrm{Mg}$ elements are enhanced by a factor of 3.2, and $\mathrm{Ne}, \mathrm{O}$ maintain the photospheric abundances of Asplund et al. (2009). The points in Fig. 1 (top) are plotted at the effective temperature

$$
T_{\text {eff }}=\int G(T) \operatorname{DEM}(T) T \mathrm{~d} T / \int G(T) \operatorname{DEM}(T) \mathrm{d} T
$$

which is an average temperature more indicative of where a line is formed. This is often quite different than $T_{\max }$, the temperature where the $G(T)$ of a line has a maximum. The bottom plot of Fig. 1 shows the corresponding values at $T_{\max } . T_{\text {eff }}$ is nearly 3 MK for all the Ne IX, Fe XVII, O VIII lines, as detailed in Table 2, where more information is provided. Note that the Fe XVII lines have $T_{\max }=5.6 \times 10^{6} \mathrm{~K}(\log T=6.8)$. When the DEM has a steep slope or the lines are significantly blended, $T_{\text {max }}$ can be quite different than $T_{\text {eff }}$.

The $16.0 \AA$ line is often a blend of O VIII and Fe XVIII, as discussed in the Fe XVIII benchmark paper (Del Zanna 2006). However, in the observations considered here the Fe XVIII contribution is small, so the observed intensity can be used to constrain
Table 2. Observed and predicted radiances for the Parkinson (1975) AR observation.

\begin{tabular}{|c|c|c|c|c|c|c|c|}
\hline$\lambda_{\mathrm{obs}}$ & $I_{\mathrm{obs}}$ & $T_{\max }$ & $T_{\mathrm{eff}}$ & $R$ & Ion & $\lambda_{\exp }$ & $r$ \\
\hline \multirow[t]{2}{*}{9.18} & 0.117 & 6.81 & 6.51 & 1.08 & $\mathrm{Mg}$ XI & 9.169 & 0.89 \\
\hline & & & & & $\operatorname{Mg} X$ & 9.182 & 0.06 \\
\hline 10.66 & 0.021 & 7.31 & 6.52 & 0.90 & Fe XVII & 10.657 & 0.99 \\
\hline \multirow[t]{2}{*}{11.01} & 0.023 & 7.01 & 6.49 & 1.18 & Ne IX & 11.000 & 0.52 \\
\hline & & & & & $\mathrm{NaX}$ & 11.003 & 0.48 \\
\hline 11.14 & 0.057 & 6.80 & 6.52 & 0.86 & Fe XVII & 11.129 & 1.00 \\
\hline 11.26 & 0.068 & 6.80 & 6.52 & 1.02 & Fe XVII & 11.250 & 0.99 \\
\hline 11.55 & 0.052 & 6.63 & 6.49 & 0.90 & Ne IX & 11.547 & 0.99 \\
\hline 12.12 & 0.182 & 6.78 & 6.52 & 1.13 & Fe XVII & 12.124 & 1.00 \\
\hline \multirow[t]{2}{*}{12.14} & 0.086 & 6.76 & 6.53 & 0.98 & $\mathrm{Ne} \mathrm{X}$ & 12.137 & 0.33 \\
\hline & & & & & $\mathrm{NeX}$ & 12.132 & 0.66 \\
\hline 12.27 & 0.168 & 6.78 & 6.52 & 1.12 & Fe XVII & 12.264 & 1.00 \\
\hline 13.45 & 0.521 & 6.96 & 6.48 & 0.84 & Ne IX & 13.447 & 1.00 \\
\hline \multirow[t]{4}{*}{13.48} & 0.032 & 7.00 & 6.46 & 0.79 & Ne VIII & 13.483 & 0.10 \\
\hline & & & & & Ne VIII & 13.483 & 0.19 \\
\hline & & & & & Ne VIII & 13.491 & 0.24 \\
\hline & & & & & Ne VIII & 13.493 & 0.39 \\
\hline 13.55 & 0.163 & 6.98 & 6.47 & 0.59 & Ne IX & 13.553 & 0.94 \\
\hline 13.70 & 0.334 & 6.56 & 6.47 & 0.92 & Ne IX & 13.699 & 0.98 \\
\hline 13.82 & 0.174 & 6.80 & 6.51 & 1.01 & Fe XVII & 13.825 & 1.00 \\
\hline \multirow[t]{2}{*}{14.21} & 0.098 & 6.90 & 6.57 & 0.85 & Fe XVIII & 14.209 & 0.36 \\
\hline & & & & & Fe XVIII & 14.204 & 0.64 \\
\hline 14.37 & 0.039 & 6.90 & 6.57 & 0.56 & Fe XVIII & 14.373 & 1.00 \\
\hline 14.53 & 0.042 & 6.90 & 6.57 & 0.42 & Fe XVIII & 14.537 & 1.00 \\
\hline \multirow[t]{2}{*}{14.82} & 0.080 & 7.01 & 6.47 & 0.84 & O VIII & 14.821 & 0.33 \\
\hline & & & & & O VIII & 14.821 & 0.67 \\
\hline 15.01 & 2.6 & 6.76 & 6.51 & 1.02 & Fe XVII & 15.013 & 1.00 \\
\hline \multirow[t]{3}{*}{15.18} & 0.320 & 6.54 & 6.47 & 0.68 & O VIII & 15.176 & 0.23 \\
\hline & & & & & O VIII & 15.176 & 0.47 \\
\hline & & & & & Fe XVI & 15.169 & 0.30 \\
\hline 15.26 & 1.3 & 6.76 & 6.51 & 0.62 & Fe XVII & 15.262 & 1.00 \\
\hline \multirow[t]{2}{*}{15.45} & 0.308 & 6.75 & 6.50 & 0.76 & Fe XVII & 15.453 & 0.78 \\
\hline & & & & & Fe XVI & 15.449 & 0.20 \\
\hline \multirow[t]{4}{*}{16.00} & 0.661 & 6.87 & 6.47 & 0.91 & O VIII & 16.007 & 0.28 \\
\hline & & & & & O VIII & 16.006 & 0.57 \\
\hline & & & & & Fe XVIII & 16.005 & 0.07 \\
\hline & & & & & Fe XVII & 16.004 & 0.08 \\
\hline 16.07 & 0.066 & 6.87 & 6.56 & 1.01 & Fe XVIII & 16.072 & 1.00 \\
\hline 16.78 & 1.9 & 6.74 & 6.50 & 1.15 & Fe XVII & 16.776 & 1.00 \\
\hline 17.05 & 2.7 & 6.74 & 6.50 & 1.06 & Fe XVII & 17.051 & 0.99 \\
\hline 17.10 & 2.2 & 6.72 & 6.50 & 1.19 & Fe XVII & 17.096 & 1.00 \\
\hline \multirow[t]{4}{*}{17.40} & 0.163 & 6.50 & 6.46 & 0.70 & O VII & 17.396 & 0.40 \\
\hline & & & & & Fe XVI & 17.390 & 0.29 \\
\hline & & & & & Fe XVI & 17.399 & 0.08 \\
\hline & & & & & Fe XVI & 17.402 & 0.18 \\
\hline 17.77 & 0.113 & 6.35 & 6.42 & 0.97 & O VII & 17.768 & 1.00 \\
\hline 18.63 & 0.393 & 6.34 & 6.42 & 0.97 & O VII & 18.627 & 1.00 \\
\hline \multirow[t]{2}{*}{18.97} & 3.5 & 6.48 & 6.46 & 1.33 & O VIII & 18.973 & 0.33 \\
\hline & & & & & O VIII & 18.967 & 0.67 \\
\hline 21.60 & 3.0 & 6.32 & 6.41 & 1.13 & O VII & 21.602 & 1.00 \\
\hline 21.80 & 0.938 & 6.29 & 6.39 & 0.62 & O VII & 21.807 & 0.99 \\
\hline 22.10 & 2.5 & 6.30 & 6.39 & 0.83 & O VII & 22.101 & 1.00 \\
\hline
\end{tabular}

Notes. $\lambda_{\mathrm{obs}}(\AA)$ are the observed wavelengths, $I_{\mathrm{obs}}$ the measured radiances (photons $\mathrm{cm}^{-2} \mathrm{~s}^{-1} \operatorname{arcsec}^{-2}$ ), $T_{\max }$ and $T_{\text {eff }}$ the maximum and effective temperature (log values, in $\mathrm{K}$; see text), $R$ the ratio between the predicted and observed radiances, Ion and $\lambda_{\exp }(\AA)$ the main contributing ion, and $r$ the fractional contribution to the blend.

very accurately the $\mathrm{O} / \mathrm{Fe}$ abundance, considering that the effective temperature of this O VIII line is the same as that of some of the Fe XVII strong lines, as shown in Table 2. The other O VIII self-blend, at $18.97 \AA$, has an effective temperature slightly less. The Ne IX lines have an effective temperature in between the O VIII 16.0 and $18.97 \AA$ lines, so the Ne IX abundance can be estimated quite accurately with respect to the $\mathrm{O}$ abundance. The H-like Ne X has also an effective temperature close to Fe XVII, and confirms the $\mathrm{Ne}$ abundance obtained from Ne IX. 
This is an important result. The Mg XI 9.17 A line also has a similar effective temperature. We obtain the $\mathrm{Mg}$ abundance shown in Table 1.

The Parkinson (1977) relative abundances, if we scale the iron value to the present one (8.0 dex), were: $\mathrm{O}(8.80)$, $\mathrm{Ne}(8.00)$, $\mathrm{Mg}$ (8.00), Si (8.00), and Ni (6.75). They are slightly different (by about $25 \%$ ) from the values obtained here for $\mathrm{O}, \mathrm{Ne}$ and $\mathrm{Mg}$. The Ni value is $65 \%$ higher than the value obtained in Del Zanna (2013b). We note that the few Ni XIX lines in the Parkinson (1975) spectrum are formed at high temperatures, where there are no firm constraints from iron lines. On the other hand, the Del Zanna (2013b) results are based on a few strong lines from Ni XV, Ni XVI, Ni XVII all formed around 3 MK, where good constraints from iron lines are available. So, the nickel abundances obtained by Del Zanna (2013b) are reliable, although we note that the atomic data for these nickel ions need some improvement.

\section{SMM FCS}

The SMM FCS database was searched for quiescent AR observations. The types of observations considered here typically started with a monochromatic image of an active region. The instrument was then pointed at the brightest region (in the AR core), where the FCS scanned the 13-20 A region. Each scan lasted about $10 \mathrm{~min}$. We obtained a long list of AR observations, summarised in Table 3. They include all those listed by previous authors, e.g. Schmelz et al. (1996), Phillips et al. (1997), and Saba et al. (1999).

We searched the count rates in the SMM bent crystal spectrometer (BCS) Ca XVIII/Ca XIX channel for signs of variability. Since FCS scans across wavelength with time, it is in fact necessary to ensure that as little solar variability is present during each scan as possible, because we are using for the diagnostics intensities of lines observed at different wavelengths (times). We are also interested in quiescent AR core observations, i.e. not in hot loops formed after obvious small flaring events, which are quite common in active regions.

We found that many AR observations in our list were not actually taken during quiescence, and so we discarded them. Figure A. 1 shows the BCS count rates for the observations which we discarded in our analysis. In addition, many other observations had a very low signal, or did not have any BCS data associated with them, and so these datasets have also been discarded. The observations selected for further analysis are listed in Table 3, and the respective BCS count rates shown in Fig. 2.

Observations of six active regions remained for further analysis. To have an idea of the type of active regions selected by this process, we searched for ground-based observations. Figure 3 shows Kitt Peak Vacuum Telescope (KPVT) magnetograms of these six ARs, obtained from the Virtual Solar Observatory. There is clearly a variety of active regions, some more dispersed, some more compact, some younger, some older.

NOAA 4729 was a fairly extended region with small sunspots, and probably at least a week old, when it was observed by SMM FCS near central meridian on 1986 May 20. NOAA 4731 was observed on 1986 May 21 about three days after its appearance at the east limb. It was a fairly large AR with many small sunspots, probably a few days old. It was observed again on May 23 and 24 when it was quiescent. NOAA 4790 was a fairly large active region with a large sunspot group. It was observed on 1987 Apr. 11. It appeared on the east limb on Apr. 6, hence it was at least a week old. It was observed again on Apr. 13 and 14 as it approached the central meridian.
Table 3. List of the SMM FCS AR observations considered here.

\begin{tabular}{|c|c|c|c|c|}
\hline $\begin{array}{l}\text { Date } \\
\text { (times, UT) }\end{array}$ & NOAA & $\mathrm{BCS}$ & Neon & FIP \\
\hline 1986 Feb. 14 & 4713 & variable & & \\
\hline $\begin{array}{l}\text { 1986 May } 20 \\
(00: 33,00: 43,00: 53)\end{array}$ & 4729 & quiet & High & 3.2 \\
\hline $\begin{array}{l}1986 \text { May } 18 \\
1986 \text { May } 21 \\
(14: 16,14: 26,14: 35)\end{array}$ & $\begin{array}{l}4731 \\
4731\end{array}$ & $\begin{array}{l}\text { variable } \\
\text { quiet }\end{array}$ & Low & $5 ?$ \\
\hline 1986 May 23 & 4731 & quiet & & \\
\hline $\begin{array}{l}\text { 1986 May } 24 \\
(05: 03,05: 13,05: 22,05: 32)\end{array}$ & 4731 & quiet & Low & 3.2 \\
\hline 1986 Feb. 14 & 4713 & variable & & \\
\hline $\begin{array}{l}\text { 1986 May } 20 \\
(00: 33,00: 43,00: 53)\end{array}$ & 4729 & quiet & High & 3.2 \\
\hline $\begin{array}{l}1986 \text { May } 18 \\
1986 \text { May } 21 \\
(14: 16,14: 26,14: 35)\end{array}$ & $\begin{array}{l}4731 \\
4731\end{array}$ & $\begin{array}{l}\text { variable } \\
\text { quiet }\end{array}$ & Low & $5 ?$ \\
\hline $\begin{array}{l}1986 \text { May } 23 \\
1986 \text { May } 24 \\
(05: 03,05: 13,05: 22,05: 32)\end{array}$ & $\begin{array}{l}4731 \\
4731\end{array}$ & $\begin{array}{l}\text { quiet } \\
\text { quiet }\end{array}$ & Low & 3.2 \\
\hline $\begin{array}{l}1986 \text { Jul. } 13 \\
1986 \text { Jul. } 14\end{array}$ & $\begin{array}{l}4736 \\
4736\end{array}$ & $\begin{array}{l}\text { no } \\
\text { variable }\end{array}$ & & \\
\hline $\begin{array}{l}1987 \text { Apr. } 9 \\
1987 \text { Apr. } 11 \\
(22: 32,22: 41,22: 51)\end{array}$ & $\begin{array}{l}4790 \\
4790\end{array}$ & $\begin{array}{l}\text { variable } \\
\text { quiet }\end{array}$ & Std. & 3.2 \\
\hline $\begin{array}{l}1987 \text { Apr. } 13 \\
(01: 14,01: 24,01: 33,01: 43)\end{array}$ & 4790 & quiet & Std. & 3.2 \\
\hline & 4790 & quiet & Std. & 3.2 \\
\hline 1987 Apr. 15 & 4790 & variable & & \\
\hline 1987 Apr. 16 & 4790 & no/variable & & \\
\hline 1987 Apr. 17 & 4790 & variable & & \\
\hline 1987 Apr. 18 & 4790 & variable & & \\
\hline 1987 Apr. 19 & 4790 & variable & & \\
\hline $\begin{array}{l}1987 \text { May } 22 \\
1987 \text { May } 26 \\
1987 \text { May } 29\end{array}$ & $\begin{array}{l}4811 \\
4811 \\
4811\end{array}$ & $\begin{array}{l}\text { variable } \\
\text { variable } \\
\text { variable }\end{array}$ & & \\
\hline $\begin{array}{l}\text { 1987 Nov. } 27 \\
(16: 25,16: 35,16: 45,16: 54)\end{array}$ & 4891 & quiet & Low & 5 \\
\hline 1987 Nov. 29 & 4891 & variable & & \\
\hline 1987 Dec. 6 & 4901 & variable & & \\
\hline 1987 Dec. 7 & 4901 & no & & \\
\hline 1987 Dec. 8 & 4901 & no & & \\
\hline 1987 Dec. 9 & 4901 & no & & \\
\hline 1987 Dec. 10 & 4901 & no & & \\
\hline $\begin{array}{l}1987 \text { Dec. } 11 \\
(2: 18,2: 28,2: 38,2: 48,2: 58)\end{array}$ & 4901 & quiet & $?$ & 3.2 \\
\hline $\begin{array}{l}\text { 1987 Dec. } 11 \\
(10: 04-11: 04)^{\dagger}\end{array}$ & 4901 & variable & & \\
\hline $\begin{array}{l}\text { 1987 Dec. } 13 \\
(09: 16,09: 26,09: 36,09: 46)\end{array}$ & 4906 & quiet & Std. & 3.2 \\
\hline 1987 Dec. 15 & 4906 & variable & & \\
\hline 1987 Dec. 16 & 4906 & variable & & \\
\hline 1987 Dec. 18 & 4906 & variable & & \\
\hline 1987 Dec. 20 & 4906 & no & & \\
\hline
\end{tabular}

Notes. The dates (and the start times below) of the FCS observations that were selected and averaged for this analysis are shown. ${ }^{(\dagger)}$ This observations was not selected. The second column indicates the NOAA number. The third column indicates the nature of the count rates in the SMM BCS Ca XIX channel. The fourth column indicates whether the neon abundance was found to be high, standard (Std.), or low by Schmelz et al. (1996). The last column shows our measured FIP enhancement of iron with respect to neon and oxygen, relative to the Asplund et al. (2009) photospheric abundances, and assuming that neon and oxygen do not vary.

However, during the later observations, a lot of variability was present. NOAA 4891 appeared small and young and was at the 

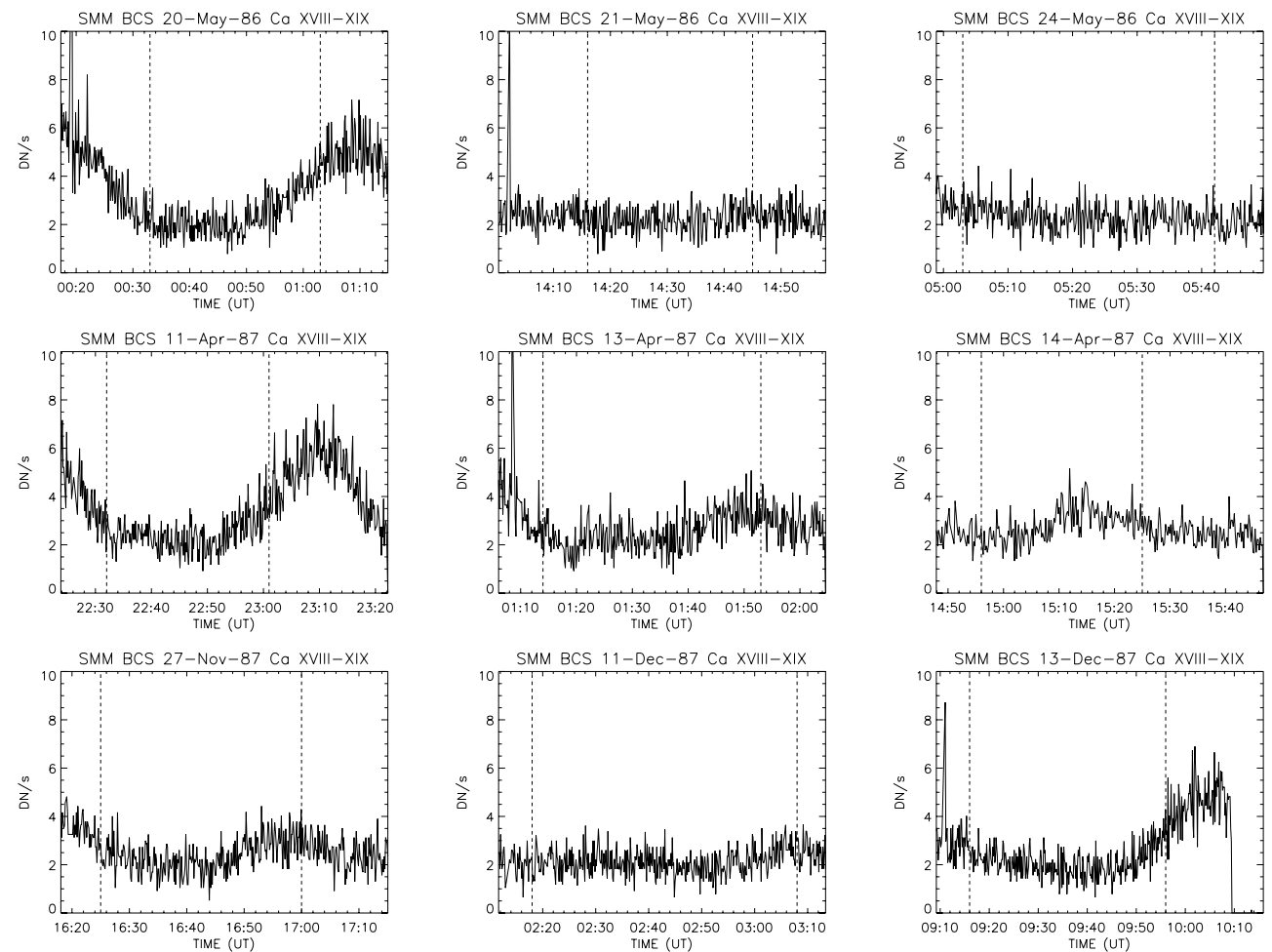

Fig. 2. Light curves in the SMM BCS Ca XIX channel (data are count rates) for the selected active regions cores analysed here. The dashed lines indicate the start and end times of the FCS observations selected for the DEM analysis.
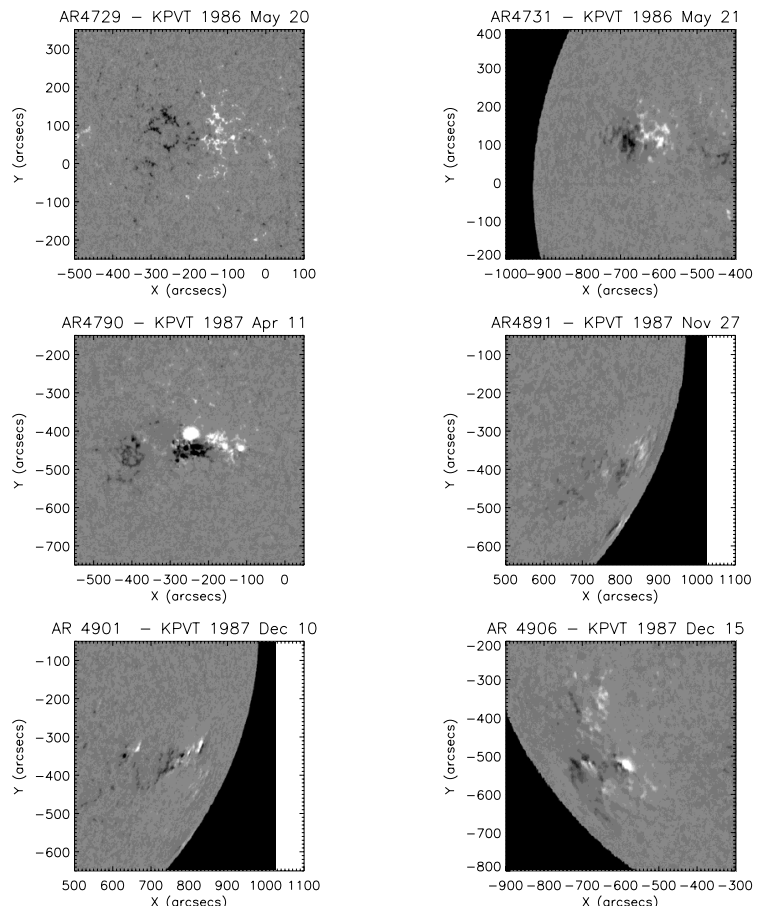

Fig. 3. KPVT magnetograms for the selected active regions analysed here.

eastern limb on 1987 Nov. 17. It was observed on Nov. 27 and 29 when it was near and at the west limb, hence when it was over 10 days old. NOAA 4901 appeared in the KPVT magnetograms of 1987 Dec. 3 near the Sun centre. It was observed with the FCS on Dec. 6-11. Unfortunately, no BCS observations for all these dates are available, except for Dec. 6 and 11. On Dec. 6,

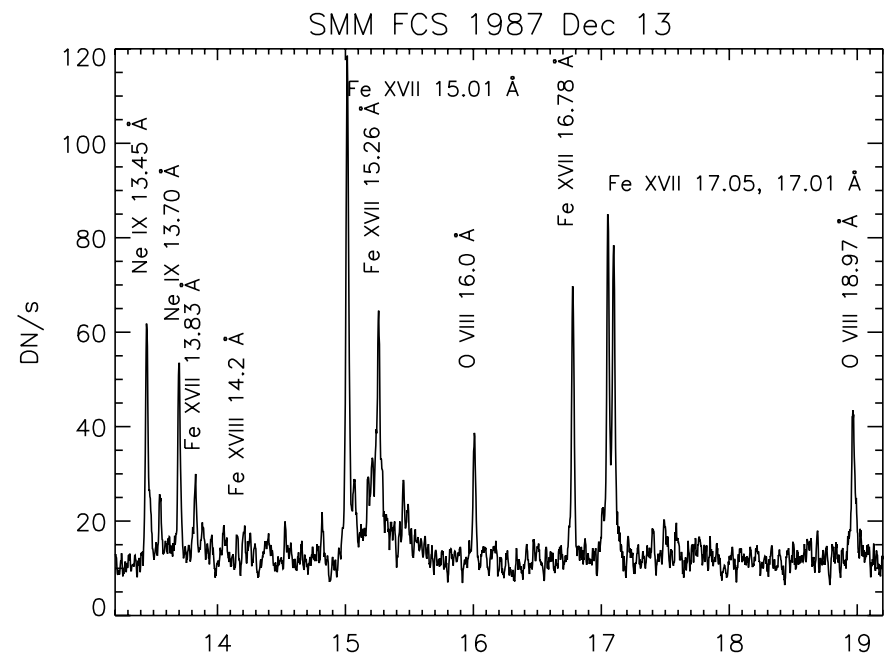

Fig. 4. SMM/FCS AR spectrum of 1987 Dec. 13.

the BCS calcium channel shows signs of strong variability. On Dec. 11, the AR was near the west limb. There are two sets of FCS raster observations, but the BCS count rates are low and the FCS data are very noisy. Finally, NOAA 4906 was observed on 1987 Dec. 13 at the SE limb. It had a fairly large sunspot, and seemed young. It was observed later, during its meridian passage, on Dec. 15, 16, 18, and 20, but the BCS calcium channel showed strong variability on those dates.

For each SMM FCS observation selected, successive spectra taken during quiescence were averaged, to increase the signal-tonoise ratio $(\mathrm{S} / \mathrm{N})$, during the time intervals displayed in Table 3 and Fig. 2. One of the best spectra in terms of $\mathrm{S} / \mathrm{N}$ was taken on 1987 Dec. 13, and is shown in Fig. 4. 
G. Del Zanna and H. E. Mason: Abundances and temperatures of active region cores

Table 4. Line intensities of the SMM FCS AR observations analysed.

\begin{tabular}{|c|c|c|c|c|c|c|c|c|c|c|}
\hline Ion & $\lambda(\AA)$ & 20-May-86 & 21-May-86 & 24-May-86 & 11-Apr.-87 & 13-Apr.-87 & 14-Apr.-87 & 27-Nov.-87 & 11-Dec.-87 & 13-Dec.-87 \\
\hline Ne IX & 13.447 & $19 \pm 6$ & $18 \pm 8$ & $16 \pm 4$ & $27 \pm 10$ & $20 \pm 6$ & $20 \pm 5$ & $12 \pm 12$ & $21 \pm 6$ & $30 \pm 8$ \\
\hline Ne IX & 13.699 & $14 \pm 4$ & $10 \pm 3$ & $11 \pm 3$ & $24 \pm 5$ & $18 \pm 4$ & $16 \pm 4$ & $13 \pm 3$ & $13 \pm 3$ & $28 \pm 6$ \\
\hline Fe XVII & 13.826 & $5 \pm 2$ & $12 \pm 3$ & $7 \pm 2$ & $7 \pm 2$ & $9 \pm 3$ & $9 \pm 2$ & $8 \pm 3$ & $13 \pm 4$ & $13 \pm 3$ \\
\hline Fe XVIII & 14.204 & $6 \pm 2$ & $12 \pm 3$ & $8 \pm 3$ & $9 \pm 3$ & $14 \pm 4$ & $16 \pm 4$ & $7 \pm 3$ & $16 \pm 4$ & $7 \pm 2$ \\
\hline Fe XVII & 15.014 & $67 \pm 14$ & $132 \pm 27$ & $116 \pm 24$ & $129 \pm 26$ & $124 \pm 25$ & $141 \pm 29$ & $117 \pm 24$ & $128 \pm 27$ & $128 \pm 26$ \\
\hline Fe XVII & 15.262 & $38 \pm 8$ & $71 \pm 15$ & $60 \pm 13$ & $64 \pm 13$ & $68 \pm 14$ & $73 \pm 15$ & $56 \pm 11$ & $55 \pm 12$ & $68 \pm 14$ \\
\hline O VIII (bl) & 16.005 & $24 \pm 5$ & $15 \pm 8$ & $17 \pm 4$ & $26 \pm 6$ & $26 \pm 6$ & $21 \pm 7$ & $20 \pm 6$ & $28 \pm 12$ & $42 \pm 10$ \\
\hline Fe XVII & 17.051 & $88 \pm 18$ & $174 \pm 35$ & $135 \pm 32$ & $146 \pm 31$ & $145 \pm 30$ & $150 \pm 33$ & $138 \pm 29$ & $184 \pm 37$ & $153 \pm 34$ \\
\hline Fe XVII & 17.096 & $109 \pm 22$ & $166 \pm 34$ & $133 \pm 28$ & $141 \pm 28$ & $118 \pm 24$ & $168 \pm 34$ & $143 \pm 30$ & $141 \pm 29$ & $171 \pm 35$ \\
\hline O VII & 18.627 & $54 \pm 23$ & $32 \pm 15$ & $25 \pm 12$ & $73 \pm 36$ & $40 \pm 15$ & $29 \pm 9$ & $14 \pm 10$ & $26 \pm 12$ & $20 \pm 13$ \\
\hline O VIII (sbl) & 18.97 & $160 \pm 45$ & $215 \pm 100$ & $180 \pm 90$ & $192 \pm 80$ & $180 \pm 75$ & $231 \pm 47$ & $209 \pm 50$ & $208 \pm 45$ & $276 \pm 105$ \\
\hline
\end{tabular}

Notes. Intensities are in phot $\mathrm{cm}^{-2} \mathrm{~s}^{-1} \operatorname{arcsec}^{-2}$.

The selected averaged spectra have then been rebinned in wavelength (to further enhance signal/ noise) and then fluxcalibrated using the software available within Solarsoft ${ }^{2}$. This includes a correction for the in-flight degradation of the instrument, which was considerable (a factor of 10) at longer wavelengths. The correction was based on the assumption of carbon deposition on the front filter (see, e.g. Saba et al. 1999). The lines were fitted with Voigt profiles using the Solarsoft cfit package (Haugan 1997). For uncertainties in the line intensities, we have considered the sum (in quadrature) of the uncertainty derived from the fitting process, plus a reasonable $20 \%$ for calibration and atomic data errors.

Intensities have then been converted to phot $\mathrm{cm}^{-2} \mathrm{~s}^{-1}$ arcsec ${ }^{-2}$ by taking into account the FCS collimator angular dimensions, which are approximately $15^{\prime \prime}$ (FWHM, equivalent to $225 \operatorname{arcsec}^{2}$ ), but are slightly dependent on the wavelength (K. Phillips, priv. comm.). The intensities of the main lines used for DEM analysis are shown in Table 4. There are some slight differences between our relative intensities and those published in the literature (normally within the uncertainties). We find good agreement with the Fe XVII line ratios published by Saba et al. (1999).

The FeXVII lines are very strong in the FCS spectra. However, they are formed around $3 \mathrm{MK}$, as discussed earlier, and also shown below. One remarkable aspect of the FCS observations of quiescent ARs is the absence of hotter emission from higher ionisation stages of iron. For example, even the strongest Fe XVIII line, the $14.20 \AA$ self-blend, is often barely visible, as seen for example in Fig. 4. This confirms the general lack of hot emission above $4 \mathrm{MK}$ in the cores of quiescent active regions.

The He-like Ne IX lines are normally observable, although often the best measurement is of the resonance Ne IX $13.45 \AA$ line. The O VIII $18.97 \AA$ and $16.00 \AA$ self-blends are often just above noise. The Mg XI line was found normally too weak to be reliable, so it has not been considered here.

We recall that Schmelz et al. (1996) used the EM loci method to find large variations in the $\mathrm{Ne}$ abundance, compared to $\mathrm{O}, \mathrm{Fe}$ and Mg. Schmelz et al. (1996) adopted a standard set of coronal abundances close to those recommended by Meyer (1985). In particular, they adopted a $\mathrm{Ne} / \mathrm{H}$ value of $3.55 \times 10^{-5}$, an $\mathrm{Fe} / \mathrm{O}$ standard abundance of 0.2 , and an $\mathrm{Fe} / \mathrm{Ne}$ abundance of 1.3 (Meyer 1985 recommended 0.16 and 1.1 respectively). These are very close to the relative abundances recommended here, 0.2 and 1.2 .

\footnotetext{
2 www.lmsal.com/solarsoft/
}

In terms of atomic data, Schmelz et al. (1996) used the Arnaud \& Rothenflug (1985) and Arnaud \& Raymond (1992) ionisation equilibria, DW data for Fe XVII and Fe XVIII, and a set of emissivities for the other ions tabulated by Mewe et al. (1985). It is not possible to reproduce exactly the Schmelz et al. (1996) results, however Fig. 5 (top) shows the EM loci curves for the SMM/FCS AR spectrum of 1987 Dec. 13 using their standard set of abundances, the Arnaud \& Rothenflug (1985) ionisation equilibria for oxygen and neon, and Arnaud \& Raymond (1992) for iron, with atomic data from CHIANTI v.4.2, which also had DW data for Fe XVII and Fe XVIII. We can see that the curves cluster around $3 \mathrm{MK}(\log T[\mathrm{~K}]=6.5)$, if one neglects the Fe XVII resonance line at $15.01 \AA$. We can therefore reproduce the Schmelz et al. (1996) result (their standard abundances for this dataset), although the same set of observations with the current atomic data show significant differences (see Fig. 5, bottom plot). The main differences are due to changes in the ion fractions for all the elements. Significant differences in the Fe XVII curves are also clear (see Del Zanna 2011 for detailed comparisons of DW and $R$-matrix calculations for this ion). The net result is a much better agreement between observations and the latest atomic data, with an isothermal temperature close to $\log T[\mathrm{~K}]=6.4$, but still similar relative abundances as those obtained with the older atomic data.

As in the previous case, we have set out to perform a full DEM analysis on all the datasets listed in Table 3, to improve on the strictly isothermal assumption. The results of the DEM analyses are summarised in Table 3. In all cases the Ne/O Asplund et al. (2009) photospheric abundance represents well the observations. The difference with the Schmelz et al. (1996) result (where the neon abundance was found to vary) are to be ascribed to the large differences in the ionisation equilibria mentioned previously, and the use of the DEM method, instead of the strictly isothermal assumption.

In most cases, we found the need to increase the $\mathrm{Fe} / \mathrm{O}$ and $\mathrm{Fe} / \mathrm{Ne}$ abundance by a factor of 3.2 , compared to the photospheric values, in order to get consistent results. This is in agreement with the previous $\mathrm{Fe} / \mathrm{Ar}$ and $\mathrm{Fe} / \mathrm{S}$ results (Del Zanna 2013b). Only in one case (1987 Nov. 27), does the Fe abundance need to be increased by a factor of 5. The 1986 May 21 observation also indicates a need for such higher FIP bias, however the spectra are very noisy, in particular for the $16.0 \AA$ line.

Figure 6 (top) shows as an example the DEM distribution for the SMM/FCS AR spectrum of 1987 Dec. 13, where the $\mathrm{Fe}$ abundance was increased by a factor of 3.2 compared to the Asplund et al. (2009) photospheric abundance (O and Ne were 


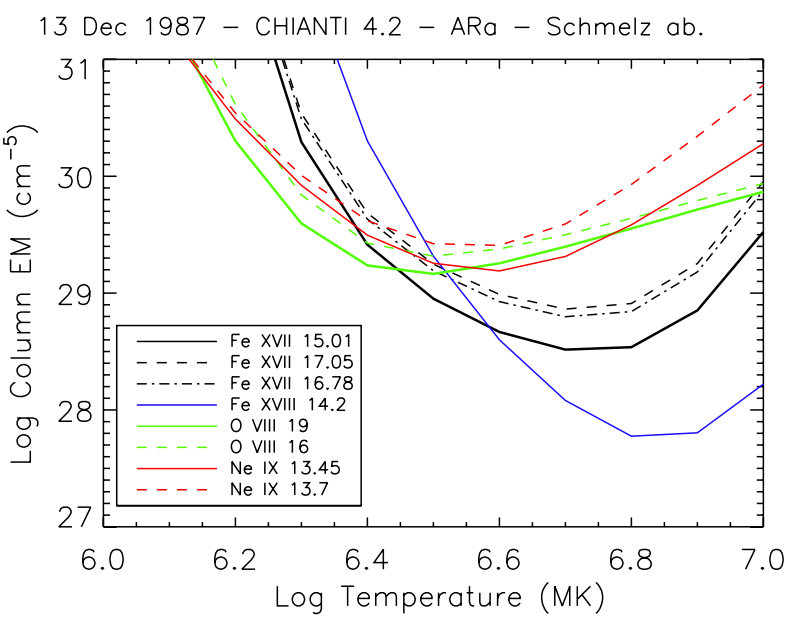

13 Dec 1987 - CHIANTI 7.1 - present ab.

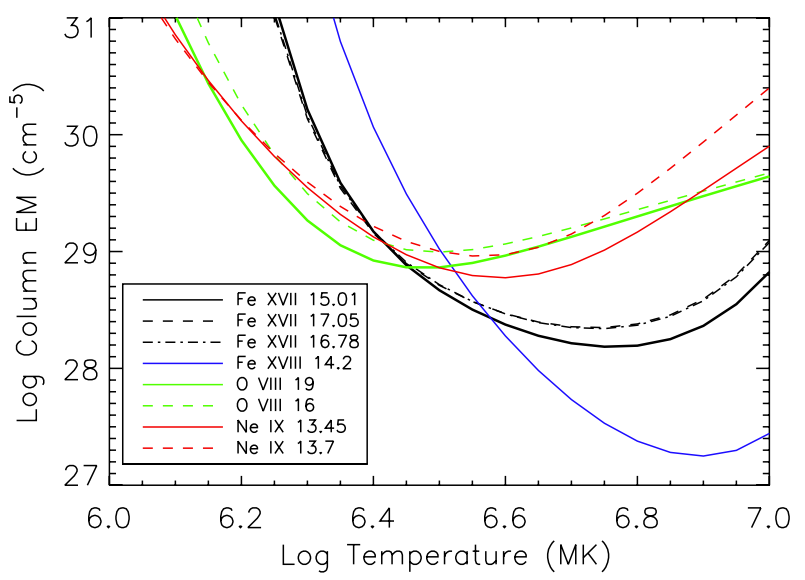

Fig. 5. EM loci curves for the SMM/FCS AR spectrum of 1987 Dec. 13. Top: with CHIANTI v.4.2, the Arnaud \& Rothenflug (1985) and Arnaud \& Raymond (1992) ion abundances, and the Schmelz et al. (1996) elemental abundances. Bottom: with the present atomic data and abundances.

kept at the Asplund et al. 2009 photospheric abundances). The $\mathrm{SMM} / \mathrm{FCS}$ data provide very good constraints in the $3 \mathrm{MK}$ to $5 \mathrm{MK}$ range. Constraints at lower temperatures are more problematic. The O VII $18.63 \AA$ line constraints lower temperatures but it is in fact barely visible. We have provided an intensity for this line, but it has an uncertainty of about $50 \%$ or more. The DEM distribution was obtained with the spline method, assuming that it has a decrease at temperatures lower than $3 \mathrm{MK}$, according to the EUV results mentioned in the introduction.

As shown in Del Zanna (2011), the Fe XVII lines are an excellent temperature diagnostic in the 3-5 MK region. The Fe XVII intensities are consistent with a sudden drop of the DEM from around $3 \mathrm{MK}$ to $5 \mathrm{MK}$ by at least 3 orders of magnitude. This is a solid and important result. In terms of emission measures, $\mathrm{EM} \simeq T^{-24}$ in the $3-5 \mathrm{MK}$ range. The lack of any emission from hotter ions provides further constraints at the higher temperatures.

We have also tested various other DEM inversion methods. As we have found in previous cases, the results of automatic inversion methods are quite sensitive to the temperature range, and depend on the temperature bin size. For well-constrained cases, the results are very similar (Del Zanna et al. 2011c; O'Dwyer et al. 2014). Figure 6 (bottom) shows as an example the DEM distribution obtained from the XRT_DEM inversion code (black histogram) and of 400 Monte Carlo XRT_DEM inversions (grey
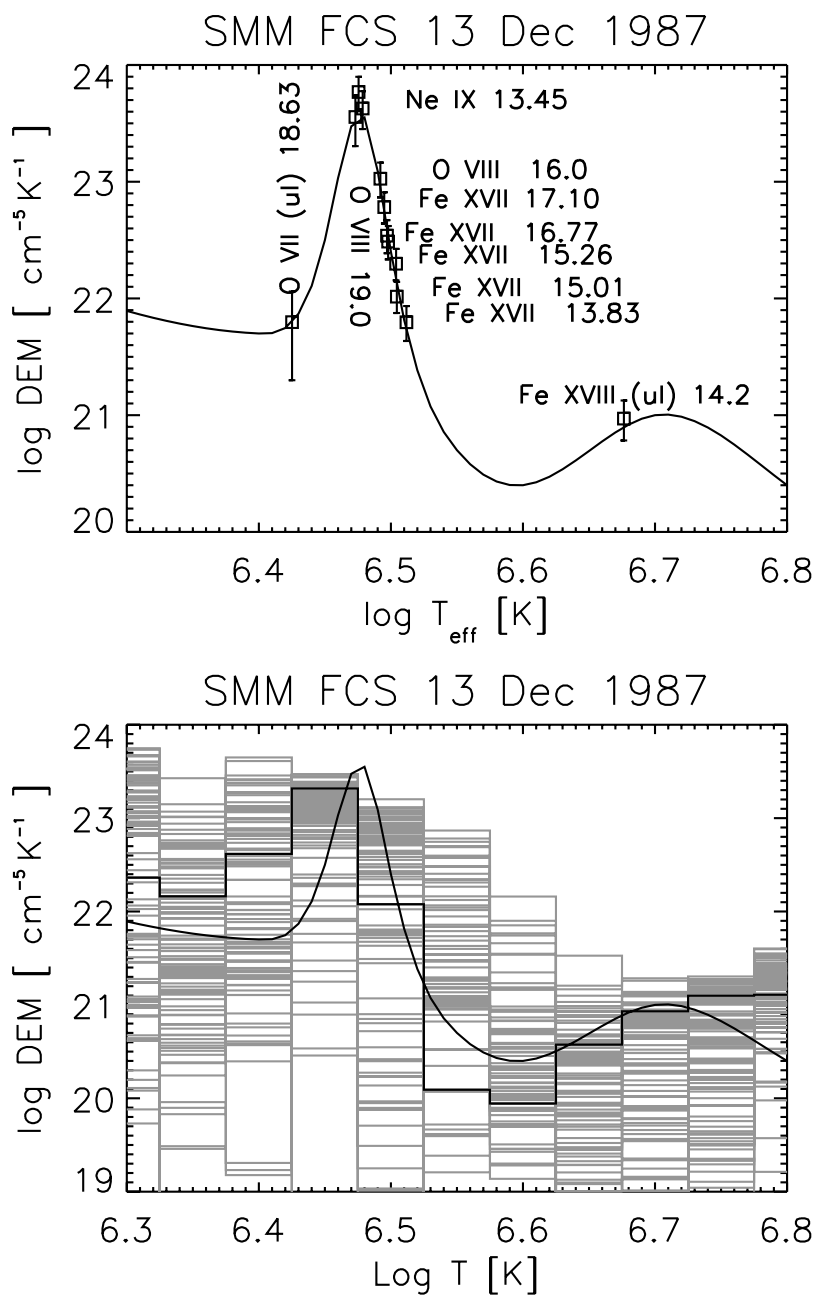

Fig. 6. Top: DEM distribution for the SMM/FCS AR spectrum of 1987 Dec. 13, with the Fe abundance increased by a factor of 3.2 compared to the Asplund et al. (2009) photospheric abundance (as Fig. 1). The points are displayed at the effective temperature. ul indicates an upper limit. The labels indicate the main ion and the wavelength in $\AA$. Bottom: same curve, with the results of the XRT_DEM inversion code (black histogram) and of the Monte Carlo XRT_DEM inversion (grey histograms).

histograms). The Monte Carlo simulations are obtained by randomly varying the input intensities within the estimated uncertainties. There is good agreement in the peak DEM and in the $\log T[\mathrm{~K}]=6.6-6.7$ range, while differences are present in the ill-constrained low- and high-temperature regions.

As in the case of the Parkinson's spectrum, the O VIII and Fe XVII have an excellent overlap in temperature, so the iron/oxygen abundance can be measured accurately. The same is true for the neon/oxygen abundance. Table 5 lists the observed and predicted radiances for the SMM FCS AR observation of 1987 Dec. 13, as in Table 2. Excellent agreement (within a relative $30 \%$ ) between observed and predicted radiances is found for all the lines, with the elemental abundances shown in Table 1, where the low-FIP Fe is enhanced by a factor of 3.2, and $\mathrm{Ne}$, O maintain the Asplund et al. (2009) photospheric abundances.

It is important to note the slight differences between the peak of the DEM $(\log T[\mathrm{~K}]=6.47)$, the isothermal temperature that can be estimated from the EM loci plot (cf. Fig. 5, about log $T[\mathrm{~K}]=6.4$ ), and the effective temperature at which most of the emission lines are formed (about $\log T[\mathrm{~K}]=6.5$ ), displayed in Table 5. The points in Fig. 6 are displayed at their effective 

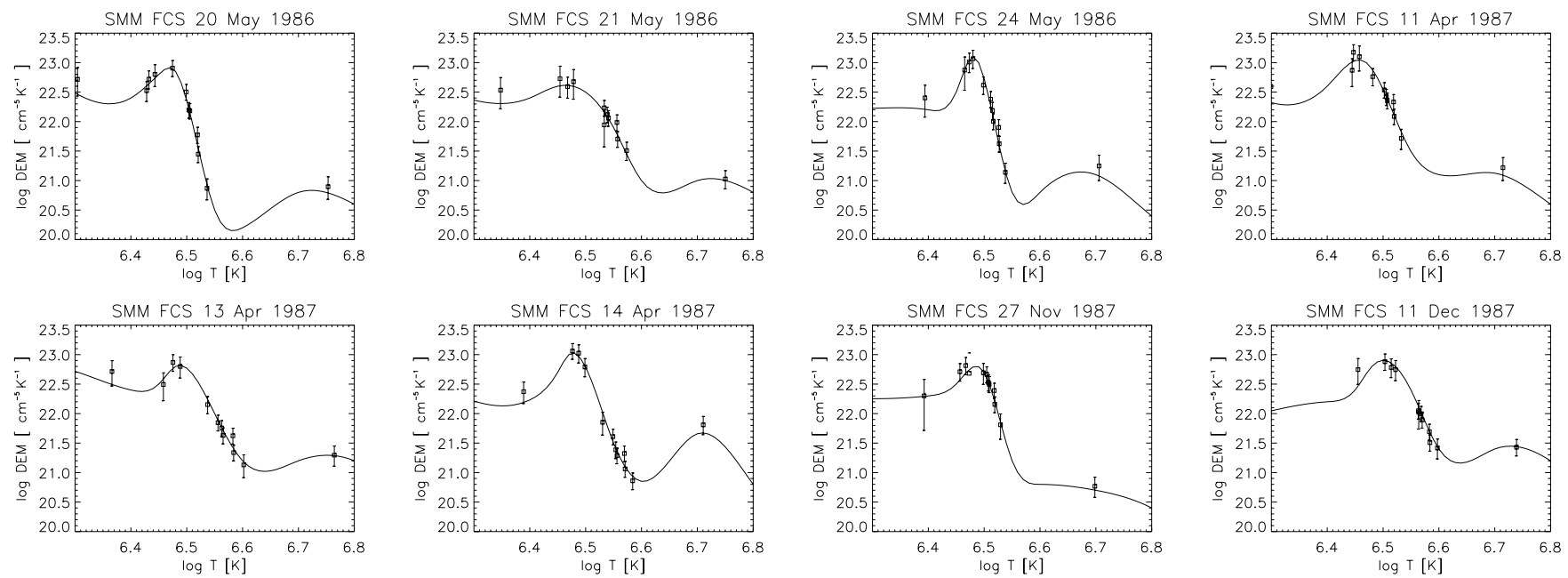

Fig. 7. DEM distributions for the active region cores analysed here, as in Fig. 6.

Table 5. Observed and predicted radiances for the SMM FCS AR observation of 1987 Dec. 13 (columns as in Table 2).

\begin{tabular}{rrrrrlrr}
\hline \hline$\lambda_{\text {obs }}$ & $I_{\text {obs }}$ & $T_{\max }$ & $T_{\text {eff }}$ & $R$ & Ion & $\lambda_{\exp }$ & $r$ \\
\hline 13.45 & 30.1 & 6.59 & 6.48 & 0.88 & Ne IX & 13.447 & 1.00 \\
13.55 & 7.7 & 6.98 & 6.48 & 0.74 & Ne IX & 13.553 & 0.96 \\
13.70 & 27.7 & 6.56 & 6.48 & 0.65 & Ne IX & 13.699 & 1.00 \\
13.83 & 12.5 & 6.79 & 6.51 & 0.86 & Fe XVII & 13.825 & 1.00 \\
14.20 & $6.8(\mathrm{ul})$ & 6.90 & 6.68 & 0.87 & Fe XVIII & 14.209 & 0.36 \\
& & & & & Fe XVIII & 14.204 & 0.64 \\
15.02 & 128.0 & 6.76 & 6.50 & 1.27 & Fe XVII & 15.013 & 1.00 \\
15.26 & 68.5 & 6.76 & 6.50 & 0.71 & Fe XVII & 15.262 & 1.00 \\
16.01 & 42.5 & 6.87 & 6.49 & 0.85 & O VIII & 16.007 & 0.28 \\
& & & & & O VIII & 16.006 & 0.56 \\
& & & & & Fe XVIII & 16.005 & 0.07 \\
& & & & & Fe XVII & 16.004 & 0.08 \\
16.78 & 116.0 & 6.74 & 6.50 & 1.15 & Fe XVII & 16.776 & 1.00 \\
17.05 & 152.9 & 6.74 & 6.50 & 1.17 & Fe XVII & 17.051 & 1.00 \\
17.10 & 171.2 & 6.72 & 6.49 & 0.93 & Fe XVII & 17.096 & 1.00 \\
18.63 & $20.1(\mathrm{ul})$ & 6.34 & 6.43 & 1.00 & O VII & 18.627 & 1.00 \\
18.97 & 276.0 & 6.48 & 6.47 & 0.99 & O VIII & 18.973 & 0.33 \\
& & & & & O VIII & 18.967 & 0.67 \\
\hline & & & & & & &
\end{tabular}

temperature, with the corresponding uncertainties, which provide a measure on the uncertainty in the DEM at the various temperatures.

The DEM distributions for the other observations are shown in Fig. 7. They are similar, with a peak around $3 \mathrm{MK}$ and a sharp drop off at higher temperatures, although the steepness at higher temperatures is not always the same. The weak Fe XVIII emission that is sometimes present requires some additional emission measure around $5 \mathrm{MK}$. In general, the FCS DEM distributions are steeper than those of Parkinson (1975). We recall that the FCS measurements were made over small angular regions (15" FWHM) near the brightest part in the AR cores, while the Parkinson (1975) observation was over a much larger area $\left(3^{\prime}\right.$ FWHM), and probably encompassed different structures. This could result in a less sharply peaked distribution.

\section{Conclusions}

Active region cores are notoriously variable, especially during the early stages, with frequent small flaring activity, as has quite clearly been shown by current XUV broad-band instruments, such as Hinode XRT and SDO AIA 94, $131 \AA$ (due to the emission from lines formed around 7-12 MK). The older SMM BCS (and, of course, Yohkoh SXT) observations of "quiescent" active regions also show frequent flaring activity. However, outside those periods of clear activity, there is very little (if any) emission above $3 \mathrm{MK}$, the peak temperature of most of the plasma. As discussed in Del Zanna (2013b), current EUV spectroscopy from Hinode EIS is limited in measuring weak emission above 3 MK. X-ray spectroscopy is needed in this regard, although as we have seen, it is limited in constraining lower temperatures below $3 \mathrm{MK}$.

We have revisited several older X-ray spectral measurements of quiescent active regions which we believe to be the best to date. The slope in the EM distribution that we found is much steeper than previous estimates, such as those of Warren et al. (2012), where values ranged between 6 and 10. For example, the strong Fe XVII lines indicate $\mathrm{EM} \simeq T^{-24}$ in the 3-5 MK range for the 1987 Dec. 13 SMM FCS observations. Often, estimating the distribution above $5 \mathrm{MK}$ is not really possible from SMM FCS observations, since only upper limits can be provided, because no measurable emission is found. In the case of the Parkinson (1975) AR observation, the EM is at least as steep as $\simeq T^{-14}$ above 3 MK.

The fact that the slope in the EM distribution above $3 \mathrm{MK}$ in quiescent active regions is extremely steep has important implications for coronal heating modelling. Models that involve impulsive heating (such as caused by nanoflares) normally require the presence of some hot plasma formed at or above $5 \mathrm{MK}$. We have found no evidence of such plasma in the quiescent cores of the active regions considered here. We should point out, however, that low densities and non-equilibrium effects can actually make such hot plasma difficult to observe (see, e.g. Reale \& Orlando 2008; Bradshaw \& Klimchuk 2011).

The first comprehensive measurement of the abundances of several elements in an AR core was recently published in Del Zanna (2013b), using Hinode EIS observations and a recent calibration (Del Zanna 2013a). The relative abundances of several key elements were obtained, including low-FIP (e.g. Fe, Si, $\mathrm{Ca}, \mathrm{Ni}$ ) and high-FIP (e.g. Ar, S). A FIP enhancement of 3.2 was found. It is interesting to see that most of the X-ray observations of AR cores we have studied here indicate the same FIP enhancement, considering the $\mathrm{Fe} / \mathrm{Ne}$ and $\mathrm{Fe} / \mathrm{O}$ relative abundances and adopting the photospheric abundances of Asplund et al. (2009) as reference values. We found similar results for five out of six 
different active regions. This is remarkable, although EUV spectroscopy from Hinode EIS of a few AR cores also showed a similar constancy (Del Zanna 2013a).

We note that our average FIP enhancement of 3.2 is slightly lower than the "average coronal value" of 4 suggested by Feldman et al. (1992), which is used extensively in the literature. We also note that different active regions structures have indicated a variability in abundances, and recommend the present abundances only for the hot core loops in quiescent active regions.

It is interesting to note that Ulysses measurements of slow solar wind (SSW) abundances have also shown on average FIP enhancements between 2 and 3 (von Steiger et al. 2000) during periods of solar activity, while the fast solar wind (FSW) streaming from coronal holes has nearly photospheric abundances (Gloeckler \& Geiss 2007). Recent measurements from the ACE spacecraft during solar maximum and minimum conditions (Lepri et al. 2013) also indicate that at minimum the differences in composition between the slow and fast wind are not large, while the SSW during solar maximum has an FIP enhancement. For example, the Fe/O increases by a factor of two, from the FSW during minimum to the SSW during maximum.

The FIP enhancements observed both in-situ, when active regions are present, and with XUV spectroscopy of their cores, suggest a possible contribution of the hot core loops to the SSW, perhaps via the interchange reconnection mechanism, proposed by Del Zanna et al. (2011a) to explain the AR coronal outflows observed by Hinode EIS (see, e.g. Del Zanna 2008b; Doschek et al. 2008). Future close-by remote sensing and in-situ observations with Solar Orbiter will shed some light into this issue.

We are also eagerly awaiting new X-ray spectroscopy with instruments such as the Marshall Grazing Incidence X-ray Spectrograph (MaGIXS; Kobayashi et al. 2011), which will be initially proposed as a sounding rocket experiment. MaGIXS will have enhanced sensitivity and spatial resolution in the important 6-24 A spectral region. This instrument, in combination with EUV observations, will be able to provide new accurate measurements of temperatures and chemical abundances in active regions.

Acknowledgements. The present work was funded by STFC (UK) through the University of Cambridge DAMTP astrophysics grant. We thank the referee for useful suggestions.

\section{References}

Aly, M. K., Evans, J. W., \& Orrall, F. Q. 1962, ApJ, 136, 956

Arnaud, M., \& Raymond, J. 1992, ApJ, 398, 394

Arnaud, M., \& Rothenflug, R. 1985, A\&AS, 60, 425

Asplund, M., Grevesse, N., Sauval, A. J., \& Scott, P. 2009, ARA\&A, 47, 481

Bradshaw, S. J., \& Klimchuk, J. A. 2011, ApJS, 194, 26

Davis, J. M., Gerassimenko, M., Krieger, A. S., \& Vaiana, G. S. 1975, Sol. Phys., 45,393

Del Zanna, G. 1999, Ph.D. Thesis, Univ. of Central Lancashire, UK

Del Zanna, G. 2006, A\&A, 459, 307

Del Zanna, G. 2008a, A\&A, 481, L69

Del Zanna, G. 2008b, A\&A, 481, L49
Del Zanna, G. 2011, A\&A, 536, A59

Del Zanna, G. 2012, A\&A, 537, A38

Del Zanna, G. 2013a, A\&A, 555, A47

Del Zanna, G. 2013b, A\&A, 558, A73

Del Zanna, G., \& Ishikawa, Y. 2009, A\&A, 508, 1517

Del Zanna, G., \& Mason, H. E. 2003, A\&A, 406, 1089

Del Zanna, G., Landini, M., \& Mason, H. E. 2002, A\&A, 385, 968

Del Zanna, G., Aulanier, G., Klein, K.-L., \& Török, T. 2011a, A\&A, 526, A137

Del Zanna, G., Mitra-Kraev, U., Bradshaw, S. J., Mason, H. E., \& Asai, A. 2011b, A\&A, 526, A1

Del Zanna, G., O’Dwyer, B., \& Mason, H. E. 2011c, A\&A, 535, A46

Dere, K. P., Landi, E., Young, P. R., et al. 2009, A\&A, 498, 915

Doschek, G. A., Warren, H. P., Mariska, J. T., et al. 2008, ApJ, 686, 1362

Evans, K., \& Pounds, K. A. 1968, ApJ, 152, 319

Feldman, U., Mandelbaum, P., Seely, J. F., Doschek, G. A., \& Gursky, H. 1992, ApJS, 81, 387

Gloeckler, G., \& Geiss, J. 2007, Space Sci. Rev., 130, 139

Haugan, S. V. H. 1997, CDS software note, 47

Ko, Y., Doschek, G. A., Warren, H. P., \& Young, P. R. 2009, ApJ, 697, 1956

Kobayashi, K., Cirtain, J., Golub, L., et al. 2011, in SPIE Conf. Ser., 8147

Landi, E., Young, P. R., Dere, K. P., Del Zanna, G., \& Mason, H. E. 2013, ApJ, 763,86

Lemen, J. R., Title, A. M., Akin, D. J., et al. 2012, Sol. Phys., 275, 17

Lepri, S. T., Landi, E., \& Zurbuchen, T. H. 2013, ApJ, 768, 94

Liang, G. Y., \& Badnell, N. R. 2010, A\&A, 518, A64

Lodders, K., Palme, H., \& Gail, H.-P. 2009, Landolt Börnstein, 712

Mason, H. E. 1975, MNRAS, 171, 119

Mason, H. E., Landi, E., Pike, C. D., \& Young, P. R. 1999, Sol. Phys., 189, 129

McKenzie, D. L., \& Feldman, U. 1992, ApJ, 389, 764

Mewe, R., Gronenschild, E. H. B. M., \& van den Oord, G. H. J. 1985, A\&AS, 62, 197

Meyer, J. 1985, ApJS, 57, 173

Mohan, A., Landi, E., \& Dwivedi, B. N. 2000, A\&A, 364, 835

Monsignori Fossi, B. C., Landini, M., Thomas, R. J., \& Neupert, W. M. 1994, Adv. Space Res., 14, 163

O’Dwyer, B., Del Zanna, G., Mason, H. E., et al. 2011, A\&A, 525, A137

O’Dwyer, B., Del Zanna, G., \& Mason, H. E. 2014, A\&A, 561, A20

Parkinson, J. H. 1975, Sol. Phys., 42, 183

Parkinson, J. H. 1977, A\&A, 57, 185

Patsourakos, S., \& Klimchuk, J. A. 2009, ApJ, 696, 760

Phillips, K. J. H., Greer, C. J., Bhatia, A. K., et al. 1997, A\&A, 324, 381

Reale, F. 2010, Liv. Rev. Sol. Phys., 7, 5

Reale, F., \& Orlando, S. 2008, ApJ, 684, 715

Rosner, R., Tucker, W. H., \& Vaiana, G. S. 1978, ApJ, 220, 643

Saba, J. L. R., \& Strong, K. T. 1993, Adv. Space Res., 13, 391

Saba, J. L. R., Schmelz, J. T., Bhatia, A. K., \& Strong, K. T. 1999, ApJ, 510, 1064

Schmelz, J. T., \& Pathak, S. 2012, ApJ, 756, 126

Schmelz, J. T., Saba, J. L. R., Ghosh, D., \& Strong, K. T. 1996, ApJ, 473, 519

Schmelz, J. T., Reames, D. V., von Steiger, R., \& Basu, S. 2012, ApJ, 755, 33

Strong, K. 1978, Ph.D. Thesis, University College London, UK

Tripathi, D., Klimchuk, J. A., \& Mason, H. E. 2011, ApJ, 740, 111

von Steiger, R., Schwadron, N. A., Fisk, L. A., et al. 2000, J. Geophys. Res., 105, 27217

Waljeski, K., Moses, D., Dere, K. P., et al. 1994, ApJ, 429, 909

Warren, H. P., Ugarte-Urra, I., Doschek, G. A., Brooks, D. H., \& Williams, D. R. 2008, ApJ, 686, L131

Warren, H. P., Winebarger, A. R., \& Brooks, D. H. 2012, ApJ, 759, 141

Widing, K. G., \& Feldman, U. 1993, ApJ, 416, 392

Winebarger, A. R., Schmelz, J. T., Warren, H. P., Saar, S. H., \& Kashyap, V. L. 2011, ApJ, 740, 2

Withbroe, G. L. 1975, Sol. Phys., 45, 301

Witthoeft, M. C., Badnell, N. R., Del Zanna, G., Berrington, K. A., \& Pelan, J. C. 2006, A\&A, 446, 361

Young, P. R., Mason, H. E., Keenan, F. P., \& Widing, K. G. 1997, A\&A, 323, 243

Young, P. R., Del Zanna, G., Mason, H. E., et al. 2007, PASJ, 59, 857

Page 11 is available in the electronic edition of the journal at http://www . aanda. org 
G. Del Zanna and H. E. Mason: Abundances and temperatures of active region cores

\section{Appendix A: SMM BCS light curves}
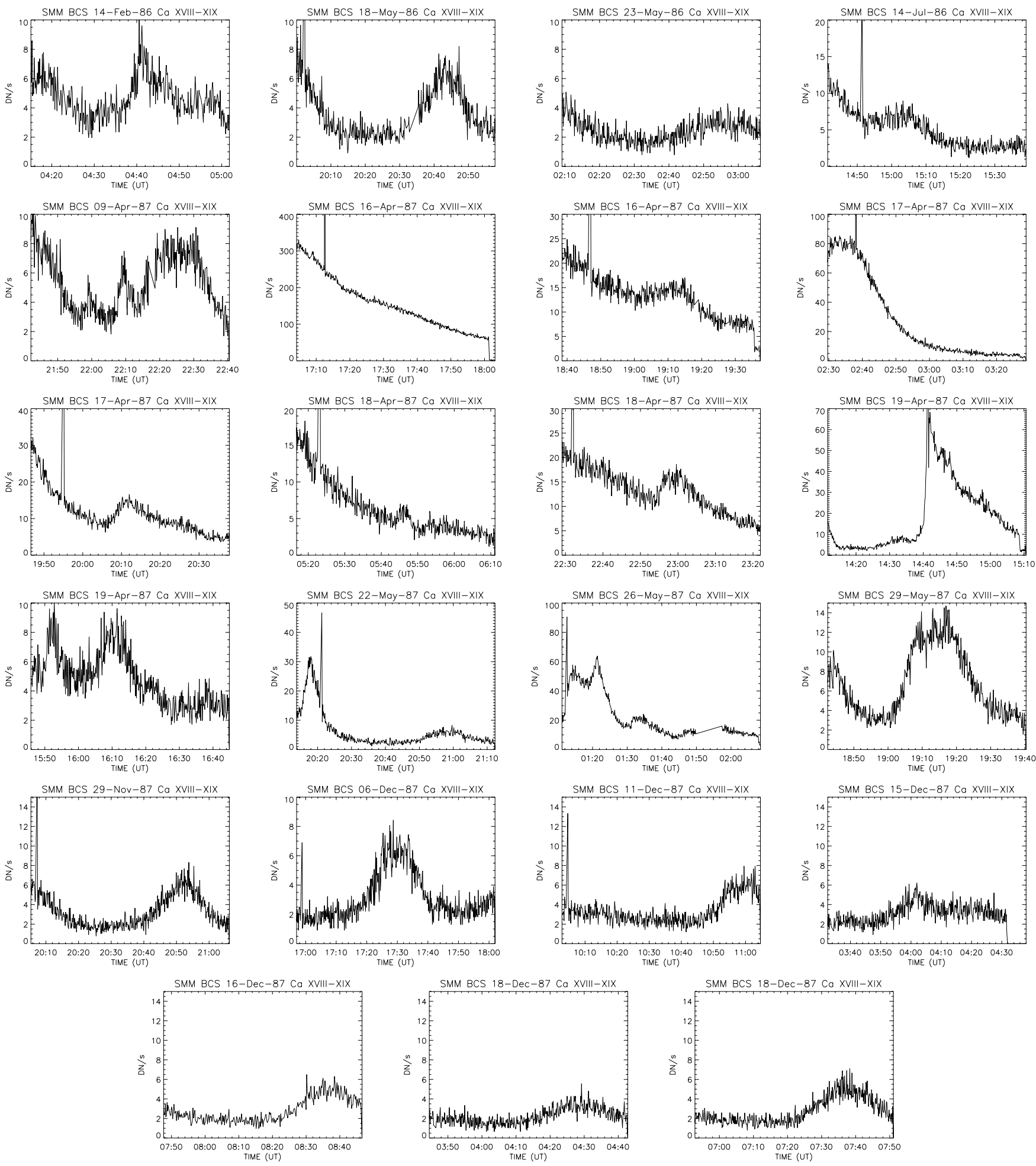

Fig. A.1. Light curves in the SMM BCS Ca XIX channel (data are count rates) for the active regions cores that were not selected for analysis. 